\title{
Um Caso de Trombocitopenia Neonatal com Neutropenia
}

\section{A Case of Alloimune Thrombocytopenia with Neutropenia}

Sónia Marques¹, Mafalda Lucas¹, Ana Rodrigues¹, Ana Rute Ferreira, Glória

Carvalhosa ${ }^{1}$, Ana Serrão Neto²

Autor Correspondente:

Sónia Marques [sonia.fonseca@jmellosaude.pt] Rua Mário Botas, 1998-018 Lisboa, Portugal

\section{RESUMO}

A trombocitopenia neonatal aloimune é a causa mais comum de trombocitopenia grave neonatal. Resulta da incompatibilidade feto-materna de antigénios plaquetares, levando à produção de anticorpos maternos e destruição das plaquetas fetais durante a gravidez.

A correlação entre aloanticorpos anti-HLA e neutropenia neonatal é extremamente rara, em parte pela reduzida expressão do sistema HLA leucócitos no recém-nascido. Uma vez que plaquetas e neutrófilos possuem sistema HLA, é possível que anticorpos HLA causem ambas neutropenia e trombocitopenia, embora sejam raras as citopenias combinadas.

Reportamos um caso clínico de trombocitopenia neonatal aloimune associada a neutropenia aloimune.

PALAVRAS-CHAVE: Neutropenia; Trombocitopenia Neonatal Aloimune

\section{ABSTRACT}

Neonatal alloimmune thrombocytopenia (NAIT) is the most common cause of severe perinatal thrombocytopenia, results from feto-maternal platelet antigen incompatibility leading to the production of maternal antibodies and destruction of fetal platelets during pregnancy. Correlation between anti-HLA alloantibodies and neonatal neutropenia is extremely rare, in part because of reduced expression of leukocyte HLAs in neonates. Because both platelets and neutrophils exhibit HLAs, it is possible for HLA antibodies to cause both neonatal alloimune neutropenia and Neonatal alloimmune thrombocytopenia. Combined alloimune cytopenias are rare. We report a case of Neonatal alloimmune thrombocytopenia associated with neutropenia alloimune.

KEYWORDS: Neutropenia; Thrombocytopenia, Neonatal Alloimmune 


\section{INTRODUÇÃO}

Trombocitopenia neonatal aloimune (TNAI) resulta da destruição das plaquetas fetais/neonatais provocada por aloanticorpos plaquetários maternos dirigidos contra antigénios plaquetários fetais herdados do pai. ${ }^{1} \mathrm{~A}$ incidência é de 1/1000-1500 gestações. A recorrência nas gravidezes seguintes é de 75\% - 90\%. ${ }^{2}$

Os anticorpos maternos mais frequentes são do tipo HPA-1a, HPA-5b e HPA-15b. 3,4 Na raça caucasiana mais de $75 \%$ dos casos de TNAI devem-se aos anticorpos HPA-1a. ${ }^{2,4,5}$

Apesar de menos comum existem casos descritos de aloimunização causada por anticorpos HLA na ausência de anticorpos HPA. ${ }^{6}$

A neutropenia neonatal aloimune (NNAI) deve-se à produção de anticorpos maternos dirigidos aos antigénios fetais herdados pelo pai e inexistentes nos neutrófilos maternos; os mais frequentes são os HNA1 e HNA2. O seu diagnóstico é feito pela deteção de anticorpos anti-neutrófilos, só acontece em 50\% dos casos. ${ }^{7.8}$

A correlação entre aloanticorpos anti-HLA e neutropenia neonatal é extremamente rara, em parte pela reduzida expressão do sistema HLA leucócitos no recém-nascido. Uma vez que plaquetas e neutrófilos possuem sistema HLA, é possível que anticorpos HLA causem ambas neutropenia e trombocitopenia, embora sejam raras as citopenias combinadas. ${ }^{8,9}$

\section{CASO CLÍNICO}

Recém-nascido (RN), sexo feminino, raça caucasiana, admitido na Unidade de Cuidados Especiais ao RN por petéquias no abdómen e membros inferiores, sem outros sintomas ou sinais de doença.

Mãe raça caucasiana, 33 anos, primeiro filho de pais não consanguíneos, sem história de pré-eclampsia, doenças de caráter heredofamiliar, sem exposição conhecida a tóxicos químicos, medicamentos, vacinas ou radiações durante a gravidez. Sem transfusões de sangue. Parto por cesariana às 39 semanas de gestação. Peso ao nascer - 2920 g. Índice de Apgar 9-10 respetivamente ao primeiro minuto e aos cinco minutos. Na observação revelava boa vitalidade, sem hepatoesplenomegalia, sem dismorfias ou outras alterações para além das petéquias no abdómen e membros inferiores.

Analiticamente revelava no primeiro dia de vida leucócitos 3300/ $/ \mathrm{mm}^{3}$, neutrófilos $1904 / \mathrm{mm}^{3}$, hemoglobina $13,9 \mathrm{~g} / \mathrm{dL}$ e plaquetas $5000 / \mathrm{mm}^{3}$. No terceiro dia de vida, a contagem de neutrófilos era de $730 / \mathrm{mm}^{3}$. Ras- treio séptico negativo e estudo primário da coagulação normal. Pesquisa de citomegalovírus na urina negativo. Plaquetas maternas $267000 / \mathrm{mm}^{3}$.

O RN foi tratado com transfusão de plaquetas e imunoglobulina endovenosa. As plaquetas subiram para $214000 / \mathrm{mm}^{3}$. Aos 30 dias de vida tinha plaquetas $341000 / \mathrm{mm}^{3}$, leucócitos 5800/mm³ e neutrófilos $1150 / \mathrm{mm}^{3}$.

Ecografia transfontanelar sem evidência de hemorragia intracraniana.

O teste DNA para antigénios plaquetares dos pais foi negativo. A investigação imunológica materna de plaquetas revelou a presença de anticorpos maternos HLA de classe I em circulação. Não sendo possível realizar a pesquisa de anticorpos para anti-neutrófilos, presumimos a sua presença após exclusão de outras causas de neutropenia.

\section{DISCUSSÃO}

Apesar da presença de anticorpos HLA ser comum nas grávidas, raramente causam trombocitopenia aloimune com neutropenia. A trombocitopenia e neutropenia induzidas por anticorpos HLA é um diagnóstico de exclusão.

Na revisão da literatura aparecem apenas três casos descritos de trombocitopenia aloimune neonatal com neutropenia, com presença de anticorpos anti-HLA, mas só num se documentou a presença de anticorpo HLA na mãe e no RN com anticorpos HNA e HPA negativos.

\section{CONCLUSÃO}

Este caso clínico é relevante pela ocorrência simultânea de TAIN e neutropenia. A associação entre anticorpos HLA e trombocitopenia e neutropenia é um diagnóstico de exclusão e difícil de provar.

O diagnóstico precoce e correto é fundamental para tratar o RN e permitir o aconselhamento pré-natal, bem como a terapêutica numa futura gravidez.

CONFLITOS DE INTERESSE: Os autores declaram não ter qualquer conflito de interesse na realização do presente trabalho.

FONTES DE FINANCIAMENTO: Não houve qualquer fonte de financiamento na realização do presente trabalho.

CONFIDENCIALIDADE DOS DADOS: Os autores declaram ter seguido os protocolos da sua instituição acerca da publicação dos dados de doentes. 
PROTEÇÃO DE PESSOAS E ANIMAIS: Os autores declaram que os procedimentos seguidos na elaboração do presente trabalho estão em conformidade com as normas das comissões de investigação clínica e de ética, bem como da declaração de Helsínquia e da Associação Médica Mundial.

CONFLICTS OF INTEREST: The authors declare that they have no conflicts of interest.

FINANCIAL SUPPORT: This work has not received any contribution, grant or scholarship.

CONFIDENTIALITY OF DATA: The authors declare that they have followed the protocols of their work center on the publication of data from patients.

PROTECTION OF HUMAN AND ANIMAL SUBJECTS: The authors declare that the procedures followed were in accordance with the regulations of the relevant clinical research ethics committee and with those of the Code of Ethics of the World Medical Association (Declaration of Helsinki).

\section{REFERÊNCIAS}

1. Fernandes CJ. Causes of neonatal thrombocytopenia. UpToDate. [consultado Jan 2017]. Disponível em: http://www.uptodate.com.

2. Holzhauer S, Zieger B. Diagnosis and Management of neonatal thrombocytopenia. Semin Fetal Neonatal Med. 2011;16:30510.

3. Fernández KS, Alarcón P. Nenatal thrombocytopenia. NeoReviews. 2013;14:e74-e81.

4. Symington A, Paes B. Fetal and neonatal alloimmune thrombocytopenia: harvesting the evidence to develop a clinical approach to management. Am J Perinatol. 2011;28:137-44.

5. Martin RJ, Fanaroff AA, Walsh MC. Neonatal-Perinatal Medicine: Diseases of the Fetus and Infant. $9^{\text {th }}$ ed. Amsterdam: Elsevier Mosby. 2011

6. Taaning E. HLA antibodies and fetomaternal alloimmune thrombocytopenia: Myth or meaningful? Transfus Med Rev. 2000;14:275-80.

7. Maheshwari A, Christensen RD, Calhoun DA. Immune neutropenia in the neonate. Adv Pediatr. 2002;49:317-39.

8. Marin L, Torio A, Muro M, Fernandez-Parra R, Minguela A, Bosch V, et al. Alloimmune neonatal neutropenia and thrombocytopenia associated with maternal anti HNA-1a, HPA-3b and HLA antibodies. Pediatr Allergy Immunol. 2005;16:279-82.

9. Hagimoto R, Koike K, Sakashita K, Ishida T, Nakazawa Y, Kurokawa $\mathrm{Y}$, et al. A possible role for maternal HLA antibody in a case of alloimmune neonatal neutropenia. Transfusion. 2001;41:615-20. 